\title{
Age-Specific Features of Soccer Players' Heart Dimensions
}

\author{
Tiiu Olm, Karin Baskin, Kadri Herde \\ School of Natural Sciences and Health, Tallinn University, Tallinn, Estonia
}

Email address:

tiiu.olm@gmail.com (T. Olm)

To cite this article:

Tiiu Olm, Karin Baskin, Kadri Herde. Age-Specific Features of Soccer Players' Heart Dimensions. American Journal of Sports Science. Vol. 5, No. 5, 2017, pp. 27-30. doi: 10.11648/j.ajss.20170505.11

Received: July 7, 2017; Accepted: September 7, 2017; Published: October 12, 2017

\begin{abstract}
This work concentrates on the analysis of the echocardiographic part of our complex investigation which determined the characteristics of work capacity, the cardio-respiratory system and the nervous system. Professional healthy male soccer players of the best clubs of Estonia $(n=236)$ aged 17 to 31 years were examined. Athletes were divided into three age groups. All tests were performed in the same training period. The statistical analysis of all characteristics (ANOVA-test, Pearson product moment correlation) allows us to conclude that the character of heart development depends on age. Soccer's sport-specific effect on heart produces an adaption where both the dilatation and the wall thickening of the heart occur moderately but not at the same time.
\end{abstract}

Keywords: Echocardiography, Soccer, Remodeling

\section{Introduction}

The athlete's heart remains the main topic of many investigations in the imaging modality and the continuing developments in technology have provided new insight into cardiac adaption in the presence of a divergent training pattern [1].

It was established that in athletes the dimensions of the heart depend on age, sex, body size, heredity as well as the character and duration of physical workload, that is, the type of sport they are engaged in. However, different authors have attached different relative importance to these factors. The most ignored determinant of heart dimensions seems to be age. In several studies average values have been calculated on the basis of a group of subjects differing in age by 10 to 20 years.

Physical training induces morphological and functional adaptions which affect all of the heart. Hemodynamic and neurohumoral stress depends on whether the muscular exercise performed was static or dynamic. Two main types of athlete's heart, a consequence of regular sport-related training, are described: eccentric myocardial hypertrophy due to load, and concentric hypertrophy due to resistanceload. Nevertheless sports-specific adaptive cardiac structural changes are still under debate. There is little known about the effect of mixed training loads. The aim of this study is to determine the effect of intermittent high intensity exercise of moderate duration on the cardiac characteristics of soccer players of different ages.

\section{Materials and Methods}

\subsection{Materials}

The subjects were 236 players of the best soccer clubs of Estonia. The athletes were evaluated at the same time (at the onset of the seasonal training period). All players, defenders and forwards, were healthy, free of cardiovascular diseases or any kind of structural anomalies. Athletes were divided into three age groups. The first group $(n=146)$ consisted of players aged 17 to 21 (average age $19.05 \pm 1.22$ years), the second group $(n=64)$ contained players aged 22 to 26 years (average age $23.6 \pm 1.27$ years) and the third group $(n=26)$ contained players aged 27 to 31 years (average age $29.1 \pm 1.28$ years). All groups of athletes maintained a similar training modality.

\subsection{Methods}

The test battery included anthropometrics, ECG and treadmill test; BLa concentration, VO2 max and the anaerobic threshold were recorded. The body surface area (BSA) was obtained using the formula of DuBois and DuBois for BSA [2]. All the data, reported as mean and SD, were indexed for the body surface area. The echocardiographic study was conducted by an experienced cardiologist using an 
echocardiograph GE Vivid E/9. Relative Wall Thickness (RWT) was determined as the ratio of wall thickness and end-diastolic diameter. The RWT value of 0.40 was considered the cut-off point. The ANOVA-Test was used. Statistical significance was assumed at $\mathrm{P}<0.05$. Student's t-test was conducted post hoc and reported, as indicated by significant ANOVA results. Pearson product moment correlation coefficients were calculated.

\section{Results and Discussion}

\subsection{Results}

The main results are given in the Table 1. All the data are within the normal range.

Table 1. Means of Three Age Groups.

\begin{tabular}{llll}
\hline MEANS & GROUP 1 $(\mathbf{n = 1 6 4 )}$ & GROUP 2 (n=64) & GROUP 3 (n=26) \\
\hline Age $(\mathrm{y})$ & $19.1 \pm 1.22 * *$ & $23.6 \pm 1.27 * *$ & $29.1 \pm 1.28 * *$ \\
Experience $(\mathrm{y})$ & $11.9 \pm 2.02 * *$ & $15.3 \pm 2.35 * *$ & $20.7 \pm 2.43 * *$ \\
Height $(\mathrm{cm})$ & $181.3 \pm 6.01$ & $180.6 \pm 5.79$ & $182.2 \pm 6.93$ \\
Body mass $(\mathrm{kg})$ & $74.4 \pm 6.83 *$ & $76.1 \pm 6.71 *$ & $79.70 \pm 7.48 * *$ \\
BSA $\left(\mathrm{m}^{2}\right)$ & $1.94 \pm 0.12 *$ & $1.96 \pm 0.11 *$ & $2.01 \pm 0.13 * *$ \\
Fat percentage $(\%)$ & $11.9 \pm 2.37$ & $11.30 \pm 2.29$ & $12.05 \pm 3.30$ \\
AO $(\mathrm{mm})$ & $29.1 \pm 2.46 * *$ & $30.5 \pm 2.51 *$ & $31.0 \pm 2.86 *$ \\
AO/BSA $\left(\mathrm{mm} / \mathrm{m}^{2}\right)$ & $15.0 \pm 1.40 *$ & $15.6 \pm 1.46 *$ & $15.4 \pm 1.54$ \\
LA $(\mathrm{mm})$ & $31.5 \pm 2.27 * *$ & $33.0 \pm 1.80 *$ & $33.5 \pm 3.04 *$ \\
LA/BSA $\left(\mathrm{mm} / \mathrm{m}^{2}\right)$ & $16.2 \pm 1.26 *$ & $16.9 \pm 1.03 *$ & $16.6 \pm 1.60$ \\
LVIDd $(\mathrm{mm})$ & $52.2 \pm 3.30 *$ & $53.9 \pm 3.01 *$ & $53.9 \pm 3.26$ \\
LVIDd/BSA $\left(\mathrm{mm} / \mathrm{m}^{2}\right)$ & $26.9 \pm 1.95 *$ & $27.6 \pm 1.79 * *$ & $26.8 \pm 1.68 *$ \\
IVSd $(\mathrm{mm})$ & $9.7 \pm 1.12 *$ & $9.9 \pm 1.00 *$ & $10.7 \pm 1.00 * *$ \\
IVSd/BSA $\left(\mathrm{mm} / \mathrm{m}^{2}\right)$ & $5.0 \pm 0.62 *$ & $5.1 \pm 0.66$ & $5.3 \pm 0.50 *$ \\
LVPWd $(\mathrm{mm})$ & $9.6 \pm 1.15 *$ & $9.9 \pm 1.02 *$ & $10.5 \pm 1.14 * *$ \\
LVPWd/BSA $\left(\mathrm{mm} / \mathrm{m}^{2}\right)$ & $5.0 \pm 0.62$ & $5.1 \pm 0.68$ & $5.2 \pm 0.59$ \\
\hline
\end{tabular}

** The mean is statistically different from the means of both other age groups $(\mathrm{P}>0.05)$.

* The mean is statistically different from the mean of one age group $(\mathrm{P}>0.05)$.

The age means of three groups differ with statistical significance. The same can be said about the averages of experience (training years). All average measures of the body and the heart of the different age groups are bigger in the oldest athletes (group 3). However, the increase of average values of different anthropological and heart characteristics are not similar.

\subsubsection{Anthropometrics Measures}

The variations of the height within the groups are higher than between the means of groups. However, the means of body mass differ with statistical significance. As the means of the body fat percentage of the groups don't differ, the heavier body mass of the oldest athletes can be explained by bigger, heavier muscles. The average of the body surface area (BSA) of the oldest athletes (group 3) as well as the average of their body mass differs significantly from the respective averages of the other two groups.

\subsubsection{Echocardiographic Measures}

As can be seen from the Table 1, all average measures of the heart in the oldest athletes (group 3) are greater than in the younger ones (group 1). The parts of the heart don't all enlarge similarly. The averages of aortic root (AO), left atrium (LA) and left ventricular internal diameters (LVIDd) of the older players (group 2) differ with statistical significance from the respective averages of the youngest players (group 1). However, the average thicknesses of the interventricular septum (IVSd) and the left ventricular posterior wall (LVPWd) differ significantly from the corresponding averages of the oldest athletes (group 3). Diagram 1 shows the increase in each age group's mean compared to the previous age group's mean (group 2 to group 1, and group 3 to group 2).

Correlation analysis shows that all five heart measures have strong correlations (at the 0.01 level) between themselves and body measures and moderate (at the 0.05 level) relationships with working capacity. A common pattern of correlation for all heart measures is that while there are numerous significant correlative relationships in younger age groups, they are lost or weaken in the oldest age group.

\subsection{Discussion}

\subsubsection{Aortic Root Dimensioon}

Cardiac adaption of different parts of the heart has been extensively described. However, the potential effect of top-level training on the aortic root remains less investigated. At the same time the frequency of cases of pathological dilatation of $\mathrm{AO}$ in top athletes has been increasing. For example, marked aortic dilatation occurs in the Marfan syndrome or bicuspid aortic valve, potentially leading to the lethal complications of aortic dissection and rupture [3] Several conflicting explanations for enlarged cardiac dimensions appear in the literature. D'Andrea et al. [4] concluded that the aortic root dimension was significantly greater in strength-trained athletes $(n=245)$ than in age- and gender-matched endurance athletes $(n=370)$. On the contrary, Iskander et al. [5] showed that male endurance athletes had greater AO diameters incomparison to strength athletes and controls, and strength athletes showed a non-significant trend 
towards greater dimensions at AO than controls. Pelliccia et al. [6] assessed athletes of 28 different sports disciplines $(n=1300)$ and showed that the aortic root dimension could be explained by weight, height, left ventricular mass and age, with the type of sports having a significant but lower impact. Their mean AO was greater $(32.2 \pm 2.7 \mathrm{~mm})$ than in our soccer players $(31.0 \pm 2.85 \mathrm{~mm})$ and $1.3 \%$ of the athletes had an enlarged $\mathrm{AO}$ (over $40 \mathrm{~mm}$ ). Over an 8-year follow-up period, in those male athletes the aortic root dimension increased by $2.0 \mathrm{~mm}$. In our investigation we can see a similar tendency: in the oldest group the mean aortic root dimension is by $1.9 \mathrm{~mm}$ greater than in the youngest group (the age difference between the groups is 10 years). The enlargement of the aortic root dimension with age occurs in relative indices as well: $\mathrm{AO} / \mathrm{BSA}$ increases from 15.0 to $15.4 \mathrm{~mm} / \mathrm{m}^{2}$. The greatest aortic root dimension is $37 \mathrm{~mm}$ in the two older groups. The clinical implication of these results is that marked dilatation of the aortic root in soccer players cannot be attributed to anthropometric characteristics or training loads alone, complementary medical exams should be performed.

\subsubsection{Left Atrial Dimension}

Left atrial size, having reservoir, conduit and contraction functions, has been shown to strongly correlate with diastolic dysfunction and be a predictor of cardiac diseases as atrial fibrillation, flutter and stroke. High endurance sports requiring rapid accelerations, such as soccer, result in increases in LA dimension. D’Ascenzi et al. [8], investigating competitive adolescent soccer players, found that left atrial (LA) moderate enlargement improved myocardial diastolic properties. However, considerable LA enlargement $(>45 \mathrm{~mm})$ was rare $(<2 \%)$ in athletes [7]. Our players' largest LA size was $40 \mathrm{~mm}$. Iskander et al. [9] performed a meta-analysis of the published literature to investigate into the relationship of high levels of exercise training to left atrial (LA) size and found that the LA increased in both strength- and endurance-trained elite athletes compared with controls but the largest LA diameters were reported in endurance athletes. Hoogsteen et al. [10] compared atrial dimensions in young cyclists $(17 \pm 0.2$ years $)$ and in older cyclists $(29 \pm 2.6$ years $)$ and found significally larger LA dimensions in the older athletes. In our research the mean of the LA dimension of the youngest age group differ significantly from the two older groups' means. The increase in the means of AO and LA in older age groups are very similar: the average $\mathrm{AO}$ of the group 2 is greater by $4.81 \%$ and the average LA by $4.76 \%$ than the respective means of the group 1. However, the increase in means in the oldest age group (3) is about three times smaller, just $1.64 \%$ and $1.52 \%$ (see Figure 1).

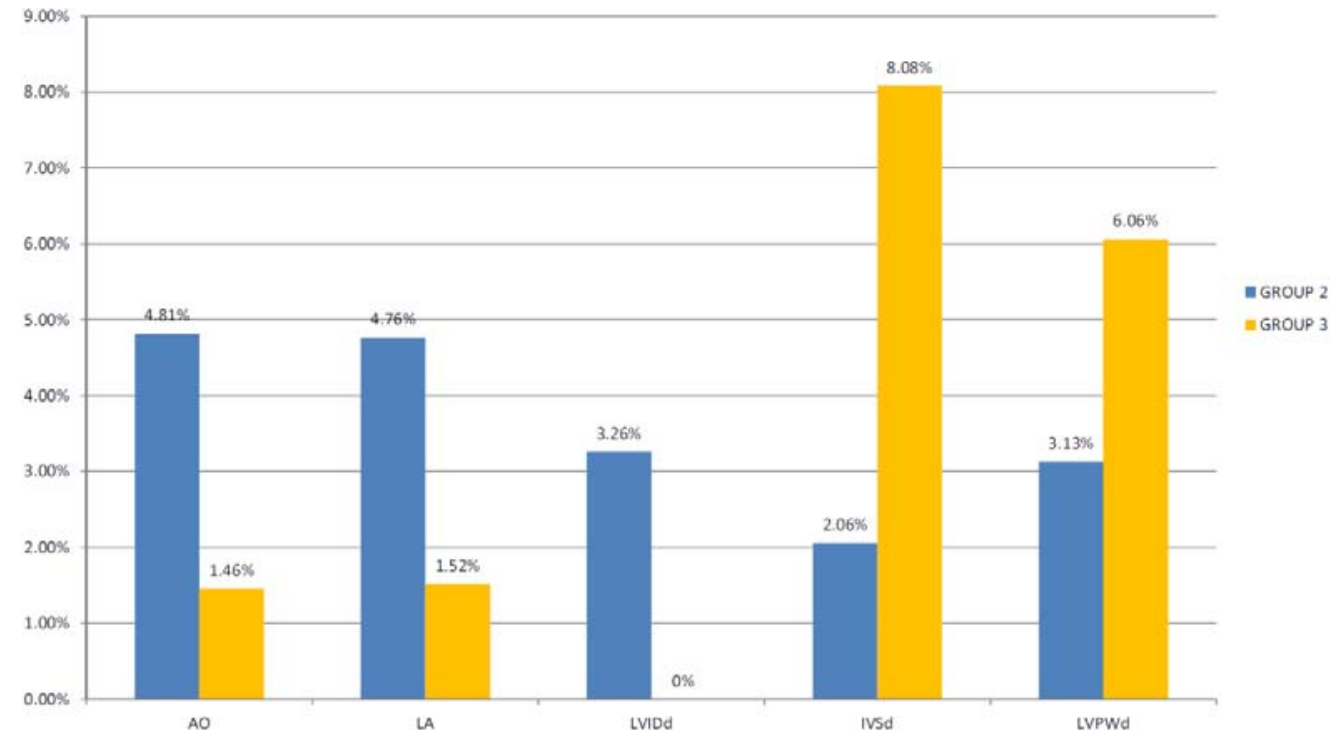

Figure 1. Increase of the Five Heart Measures (in Percentages).

\subsubsection{Left Ventricular Dimensions}

The most frequent cause of the exercise-related cardiac arrest in young athletes is hypertrophic cardiopathy (HCM). It is responsible for one third of sudden deaths [11], [12]. Therefore the discrimination between the benign physiological cardiac hypertrophy and the pathological one is obviously important. Large-sample studies have shown that a significant proportion of endurance athletes exceed the 'normal upper limit' $(55 \mathrm{~mm})$ for the left ventricular end-diastolic internal diameter [13]. Among high-level athletes from different sports, the LVID diameter was measured to be 6.0 to $7.0 \mathrm{~cm}$. This kind of eccentric hypertrophy exists in $6 \%$ of the endurance athletes [14]. We have the same percentage $(6.19 \%)$ in the older groups, but the greatest measure is just $63 \mathrm{~mm}$. The increase in the average LVIDd of group 2 in comparison with the group 1 is moderate $(3.26 \%)$ and there is no increase in the group 3 in comparison with the group 2. The average LVIDd of the two older groups is $53.9 \mathrm{~mm}$ and LVIDd/ BSA is somewhat greater in the group 2. So we can see that the increase of some heart measures (AO and LA) slows down with the age and LVIDd stops significantly dilating in the middle of twenties. However, the interventricular septum (IVSd) and the left ventricular posterior wall (LVPWd) thickness of the oldest age group 
increase rapidly ( $8.08 \%$ and $6.06 \%)$. Nevertheless, we have no players with symptoms of myocardial hypertrophy. Concentric-type myocardial hypertrophy, common to strength/power sports, is characterized as an increase in LV mass with an augmented relative wall thickness (RWT) above the arbitrary 0.42 value. Abernethy et al. [15], examining highly trained American football players, found that $23 \%$ had evidence of LV hypertrophy. The RWT for the players was $0.42 \pm 0.1$. Pluim et al. [16], comparing soccer players and adult athletes, used RWT as well. RWT-calculated as the ratio between the sum of posterior wall and interventricular septum thicknesses and left ventricular cavity dimension - is dimensionless and doesn 't depend on body size. This calculation gives a mean relative wall thickness of 0.397 for soccer players. Our mean RWT value in the oldest group was $0.40 \pm 0.04$ and in the two younger age groups just $0.37 \pm 0.05$ due to a very moderate septum and posterior wall thickness (IVSd is $9.7 \mathrm{~mm}$ to $10.7 \mathrm{~mm}$ and LVPWd is $9.6 \mathrm{~mm}$ to 10.5 $\mathrm{mm})$. The greatest value for both of them are $12 \mathrm{~mm}$, which is significantly smaller from the norm $(15 \mathrm{~mm})$.

The analysis of the contradictory data by different authors about the sport-specific influence on the heart morphology of athletes in different ages, shows as rule: 1 . The older is the investigation, the higher is the percentage of athletes having the classical type (with eccentric or concentric hypertrophy) of athlete's heart. This trend might be due to a changed in training methods during the last 20-30 years. Today most coaches use mixed exercises containing all basic (endurance, strength, speed) components. That's why the classical type of athlete's heart is rarer nowadays. 2. The younger are the athletes examined, the stronger appears to be the age-related differences in heart measures. This can explained by the developmental physiology of the heart. Rapid heart development ends after the twenties, the most changeable part of the heart in older athletes is the lef ventricle, as also show the results of this investigation.

\section{Conclusion}

We can conclude that soccer's sports-specific influence on the heart produces 'symmetric remodelling', an adaption where both the dilatation and the wall thickening of the chamber occur moderately but not at the same time. Rapid heart dilatation changes to the quick thickening of the walls in the middle of twenties. In this sensitive period the athletes need more echocardiographic observations.

Moderate, symmetric influence on the heart allows us to recommend soccer as an advisable sport for a balanced development of the heart.

\section{References}

[1] Utomi V, Oxborough D, Whyte GP, Somauroo J, Sharma S, Shave R, Atkinson G, George K. Systematic review and meta-analysis of training mode, imaging modality and body size influences on the morphology and function of the male athlete'sheart. Heart. 2013 Dec; 99(23): 1727-33.
[2] DuBois D, DuBois D F. A formula to estimate the approximate surface area if height and weight be known. Nutrition. 1989; 5: 303-11.

[3] Tadros T M, Klein M D, Shapira O M. Ascending aortic dilatation associated with bicuspid aortic valve: pathophysiology, molecular biology, and clinical implications. Circulation. 2009; 119: 880-890.

[4] D'Andrea A, Cocchia R, Riegler L, Scarafile R, Salerno G, Gravino R, Vriz O, Citro R, Limongelli G, Di Salvo G, Cuomo S, Caso P, Russo MG, Calabro R, Bossone E. Aortic root dimensions in elite athletes. Am J Cardiol. 2010; 105(11): 1629-34.

[5] Iskander A, Thompson P D. A meta-analysis of aortic root size in elite athletes. Circulation. 2013; 127: 791-798.

[6] Pelliccia A 1, Di Paolo F M, De Blasiis E, Quattrini FM, Pisicchio C, Guerra E, Culasso F, Maron B J. Prevalence and clinical significance of aortic root dilation in highly trained competitive athletes. Circulation. 2010 Aug 17; 122(7): 698-706.

[7] Pelliecia A, Maron B J, Di Paolo F M, Biffi A, Quattrini FM, Pisicchio C, Roselli A, Caselli S, Culasso F. Prevalence and clinical significance of LA remodeling in competitive athletes. J Am Coll Cardiol. 2005 Aug 16; 46(4): 690-6.

[8] D'Ascenzi F, Cameli M, Lisi M, Zaca V, Natali B, Malandrino A, Benincasa S, Causarano A, Modillo S. Left atrial remodelling in competitive adolescent soccer players. Int $\mathrm{J}$ Sport Med. 2012 Oct; 33(10): 785-801.

[9] Iskander A, Mujtaba M T, Thompson P D. Left Atrium size in Elite Athletes. JACC Cardiovasc Imaging. 2015 Jul; 8(7): 753-62.

[10] Hoogsteen J, Hoogeveen A, Schaffers H, Wijn P F, van der Wall E E. Left atrial and ventricular dimensions in highly trained cyclists. Int J Cardiovasc Imaging. 2003; 19(3): 211-217.

[11] Barry J. Maron; Antonio Pellicia. The Heart of Trained Athletes. Circulation. 2006; Volume 114: 1633-1644.

[12] Maron B. Sudden death in young athletes. N England J Med. 2003; 349: 1064-1075.

[13] Urhausen A, Monz T, Kindermann W. Echocardiographic Criteria of Physiological Left-Ventricular Hypertrophy in Combined Strength-Trained and Endurance-Trained Athletes. Int Journal Cardiac Imaging. 1997; 13: 43-52.

[14] Whyte, G. P., George, K., Sharma, S., Firoozi, S., Stephens, N., Senior, R. and McKenna, W. J. The upper limit of physiological cardiac hypertrophy in elite male and female athletes: the British experience. European Journal of Applied Physiology. 2004; 92: 592-597.

[15] Abernethy W B, Choo JK, Hutter A M Jr. Echocardiographic characteristics of professional football players. J Am Coll Cardiol. 2003; 41(2): 280-4.

[16] Pluim B M, Zwinderman A H, van der Laarse A, et al. The athlete's heart: a meta-analysis of cardiac structure and function. Circulation. 1999; 100: 336-44. 